\title{
Surface stress, surface elasticity, and the size effect in surface segregation
}

\author{
Schmid, M.; Hofer, W.; Varga, P.; Stoltze, Per; Jacobsen, Karsten Wedel; Nørskov, Jens Kehlet
}

Published in:

Physical Review B

Link to article, DOI:

10.1103/PhysRevB.51.10937

Publication date:

1995

Document Version

Publisher's PDF, also known as Version of record

Link back to DTU Orbit

Citation (APA):

Schmid, M., Hofer, W., Varga, P., Stoltze, P., Jacobsen, K. W., \& Nørskov, J. K. (1995). Surface stress, surface elasticity, and the size effect in surface segregation. Physical Review B, 51(16), 10937-10946.

https://doi.org/10.1103/PhysRevB.51.10937

\section{General rights}

Copyright and moral rights for the publications made accessible in the public portal are retained by the authors and/or other copyright owners and it is a condition of accessing publications that users recognise and abide by the legal requirements associated with these rights.

- Users may download and print one copy of any publication from the public portal for the purpose of private study or research.

- You may not further distribute the material or use it for any profit-making activity or commercial gain

- You may freely distribute the URL identifying the publication in the public portal 


\title{
Surface stress, surface elasticity, and the size effect in surface segregation
}

\author{
M. Schmid, ${ }^{*}$ W. Hofer, and P. Varga \\ Institut für Allgemeine Physik, Technische Universität Wien, A-1040 Wien, Austria \\ P. Stoltze, K. W. Jacobsen, and J. K. Nørskov \\ Center of Atomic-scale Materials Physics and Physics Department, Technical University of Denmark, DK 2800 Lyngby, Denmark
}

(Received 10 November 1994)

\begin{abstract}
Surface stress and surface elasticity of low-index fcc surfaces have been studied using effective-medium theory potentials. In addition to total-energy calculations giving stress components and elastic data for the surface as a whole, the use of artificial atoms with modified size allows us to probe the stress and elasticity of individual layers. This method of artificial atoms provides a direct way to study the contribution of atomic size to segregation in alloys as well as the driving force of reconstructions driven by surface stress. As an example, we give a qualitative explanation of the face-dependent segregation of $\mathrm{Pt}$ $\mathrm{Ni}$ alloys. We also compare results of these atomic-scale calculations with continuum elasticity.
\end{abstract}

\section{INTRODUCTION}

Fundamental thermodynamic considerations as well as the existence of surface-stress-driven reconstructions, such as the $\mathrm{Au}(111)$ herringbone reconstruction, ${ }^{1-3}$ have motivated several studies of surface stress, both by $a b$ initio methods ${ }^{4-11}$ and by semiempirical potentials. ${ }^{12-14}$ Surface elasticity, though also related to this topic, has been mainly seen from the viewpoint of surface phonons, which provide an experimental means to study some aspects of surface elasticity. ${ }^{15-17}$ Surface stress and elasticity also play an important role in segregation ${ }^{18,19}$ and in the formation of surface alloys, if the alloy components have different atomic size. Nevertheless, many thermodynamic models of segregation have used a very simplified picture of a surface in this respect.

The aim of this paper is to present a coherent study of these phenomena, which may provide the basis for discussion of many problems regarding stress and elasticity in segregation and reconstruction. As a basis of our calculations, we will use effective-medium theory (EMT) potentials, which not only give a good description of the surface properties studied here but also provide the opportunity to create artificial atoms of the same kind, but different size for the study of size effects. Numerical data will be given for palladium, which we may consider a typical fcc metal since its position in the Periodic Table is surrounded by most of those fcc metals common in surface science.

We will give a short introduction to effective-medium theory and to the form of the EMT potentials used in this article in Sec. II. We will then discuss the stress and elastic properties of a surface, i.e., the first and the second derivative of surface energy with respect to lateral strain, in Sec. III. We might call these properties total surface properties, since they are defined for the surface as a whole, without any distinction between different layers. Section IV will be devoted to extracting stress and strain contributions of different layers by the method of artificial atoms and Sec. $\mathrm{V}$ to an application of these data for segregation calculations in the Pt-Ni system.

\section{EFFECTIVE-MEDIUM THEORY}

As the basics of EMT have been described in several articles, ${ }^{20-24}$ we will only give a short introduction here. EMT is a method for total-energy calculations of solids based on density-functional theory and deriving much of its data from self-consistent calculations for the atomic species of interest immersed in a homogeneous electron gas. Due to the screening properties of the electron gas in a metal, a homogeneous electron gas of a certain density $\bar{n}$ can be considered a good approximation for the actual environment of an atom in a metal and we name the respective energy of an atom cohesive energy $E_{c}(\bar{n}) . E_{c}$ becomes a minimum at the equilibrium lattice constant; we denote the corresponding values as $E_{0}$ and $n_{0}$.

We further define the neutral sphere size $s(\bar{n})$ as the radius of a sphere where the electronic charge (of the atom and the homogeneous electron gas) and the core charge cancel. Whereas the bulk of a pure fcc metal can be closely approximated by neutral atomic spheres, for distorted geometries, surfaces, impurity atoms, etc., the atomic-sphere correction $E_{\mathrm{AS}}$ is introduced. It can be shown that $E_{\text {AS }}$ can be approximated by pair potential terms. ${ }^{20,21}$

A further correction term to the total energy is the one-electron correction $E_{1 \mathrm{el}}$. Whereas $E_{c}$ and $E_{\mathrm{AS}}$ already implicitly include the main energy contributions coming from the formation of electronic bonds, $E_{1 \mathrm{el}}$ corrects for changes of the shape of the electronic state density. $E_{1 \text { el }}$ becomes most important for lowcoordination systems ${ }^{25,26}$ and for transition metals where the partly filled $d$ band plays an important role. The one-electron term also has some influence on the elastic shear constant $c_{44}$, but this effect can be compensated for by adjusting the pairwise potentials in $E_{\mathrm{AS}}$ to yield 
TABLE I. Effective-medium parameters for $\mathrm{Pd}$.

\begin{tabular}{llll}
\hline \hline Parameter & Value & Unit \\
\hline$E_{0}$ & binding energy $\left(E_{c}\right.$ at equilibrium lattice constant) & -3.90 & $\mathrm{eV}$ \\
$n_{0}$ & electronic density at equilibrium lattice constant & 0.00688 & $\mathrm{Bohr}$ radii \\
$s_{0}$ & neutral sphere radius at equilibrium lattice constant & 2.87 & $\mathrm{Bohr}$ radii \\
$\lambda$ & curvature of $E_{c}(s)$, Eq. $(2.1)$ & 2.155 & $\mathrm{Bohr} \mathrm{radii}^{-1}$ \\
$\eta_{2}$ & decay constant, Eq. (2.3) & 1.818 & $\mathrm{Bohr}_{\mathrm{radii}}^{-1}$ \\
$V_{0}$ & preexponential, atomic-sphere correction Eq. (2.4) & 2.773 & $\mathrm{eV}$ \\
$\kappa$ & decay constant, atomic-sphere correction Eq. (2.4) & 3.107 & $\mathrm{Bohr} \mathrm{radii}^{-1}$ \\
\hline \hline
\end{tabular}

correct $c_{44}$ (see below). Since Pd has a nearly full $d$ band and the lowest coordination number examined in our study is seven (fcc 110 surface atom), we neglect the oneelectron term apart from the adjustment of the pair potential.

In the implementation used for the current work $E_{c}$ for an atom $i$ is calculated as

$$
E_{c}^{(i)}=E_{0}^{(i)} f\left(\lambda^{(i)}\left(s^{(i)}-s_{0}^{(i)}\right)\right),
$$

where $E_{0}$ and $s_{0}$ are the cohesive energy and the neutral sphere size for the fcc bulk at equilibrium lattice constant, $\lambda$ is a parameter determining the curvature of the $E_{c}(\bar{n})$ function, and $f$ is the function ${ }^{27-29}$

$$
f(x)=(1+x) \exp (-x) .
$$

The neutral sphere size for the atom $i$ under consideration

$s^{(i)}=s_{0}^{(i)}-\frac{1}{\beta \eta_{2}^{(i)}} \ln \left[\frac{1}{12 n_{0}^{(i)}} \sum_{j} n_{0}^{(j)} \exp \left[-\eta_{2}^{(j)}\left(r_{i j}-\beta s_{0}^{(j)}\right)\right]\right]$

depends on the electronic density $\bar{n}$ provided by its neighbors $j$ [the sum in Eq. (2.3)]. The decay constant $\eta_{2}$ for a given atomic species is derived from $a b$ initio calculations of the neutral sphere size as a function of density in the homogeneous electron gas, $r_{i j}$ is the interatomic distance, and $\beta=(16 \pi / 3)^{1 / 3} / \sqrt{2}$ is a dimensionless geometric parameter (the ratio between nearest-neighbor distance and neutral sphere size in an fcc lattice).

A good approximation for the atomic-sphere correction is given by

$$
\begin{array}{r}
E_{\mathrm{AS}}=-\frac{1}{2} \sum_{i} V_{0}^{(i)}\left[\frac{1}{n_{0}^{(i)}} \sum_{j(\neq i)} n_{0}^{(j)} \exp \left[-\kappa^{(j)}\left(r_{i j} / \beta-s_{0}^{(j)}\right)\right]\right. \\
-12 \exp \left[-\kappa^{(i)}\left(s^{(i)}-s_{0}^{(i)}\right)\right]
\end{array}
$$

with the parameters $V_{0}$ (positive) and $\kappa$ for each atomic species. Using these approximations the total energy

$$
E_{\mathrm{tot}}=\sum_{i} E_{c}^{(i)}+E_{\mathrm{AS}}=E_{c}+E_{\mathrm{AS}}
$$

is a sum of density- or $s$-dependent terms for each atom [ $E_{c}$ and the second exponential in Eq. (2.4)] and a pair potential [the first exponential in Eq. (2.4)]. Such a func- tional form is also used in other potentials, e.g., "glue,"30 Finnis-Sinclair, ${ }^{31}$ or embedded-atom ${ }^{32}$ potentials.

Although all element parameters could be, in principle, derived by $a b$ initio calculations, we only use $\eta_{2}$ and $n_{0}$ determined from these calculations. The other parameters are found from experimental values, i.e., the binding energy $\left(E_{0}\right)$, the lattice constant (giving $s_{0}$ ), the bulk modulus (giving $\lambda$ ), and the elastic constant $c_{44}$ (giving $\kappa)$. This approach has the advantage of higher accuracy, since the reference system or effective medium is forced to comply with the actual fcc crystal in many respects. EMT parameters for $\mathbf{P d}$ are given in Table $\mathrm{I}$.

In Eqs. (2.3) and (2.4) it has been assumed for simplicity that only nearest neighbors are counted. It has been noted previously ${ }^{33}$ that with the given functional form of the potential the anisotropy ratio of elastic constants

$$
A_{\mathrm{el}}=\frac{2 c_{44}}{c_{11}-c_{12}}
$$

equals 2 if only nearest-neighbor interactions are included. This is equivalent to the relation $c_{11}=c_{12}+c_{44} \cdot{ }^{21}$ This constraint is lifted if the interaction with furtherneighbor shells is included. In this case the two constants 12 in (2.3) and (2.4) have to be replaced by slightly larger values taking the influence of further neighbors into account. ${ }^{20,23,34}$

For Pd, experimental values of the elastic anisotropy $A_{\text {el }}$ are between 2.46 and 2.57; the effective-medium result $A_{\mathrm{el}}=2.53$ (smooth cutoff between third and fourth nearest neighbors) is in excellent agreement with experiment. Together with the determination of the EMT parameters from bulk modulus $\left(c_{11}+2 c_{12}\right) / 3$ and $c_{44}$ (see above), this ensures agreement of all three independent bulk elastic constants between experiment and EMT.

For computation of surface properties, the derivatives of surface energy have been calculated from energies of slabs with different thickness-11 and 19 layers for the more close-packed surfaces, 15 and 25 layers for the (110) surface-at different lateral strains in the order of $1 \%$. This ensures that systematic errors due to finite slab thickness and nonlinear elasticity are negligible.

\section{SURFACE STRESS AND SURFACE ELASTICITY}

\section{A. Surface stress}

In this section we will consider stress and elasticity of the surface as a whole, i.e, the first and second derivatives of the surface energy with respect to strain $\varepsilon_{\alpha \beta}$. We will 
first define the surface stress tensor, which is given as $^{4,35,36}$

$$
g_{\alpha \beta}=\frac{1}{A} \frac{\partial(A \gamma)}{\partial \varepsilon_{\alpha \beta}}=\frac{\partial \gamma}{\partial \varepsilon_{\alpha \beta}}+\gamma \delta_{\alpha \beta},
$$

where $\gamma$ is the surface energy, $A$ is the surface area, and $\delta_{\alpha \beta}$ denotes the Kronecker delta function. Here it is important to note that the surface energy $\gamma$ must be defined as energy per actual (strained) surface area $A$. For a liquid, a change of surface area necessarily implies moving additional atoms to the surface, keeping the atomic density (per area) and structure of the surface and hence the surface energy $\gamma$ unchanged. Therefore, $\partial \gamma / \partial \varepsilon_{\alpha \beta}=0$ and the surface stress tensor $g_{\alpha \beta}$ of a liquid can be replaced by a scalar $g$, which is equal to the surface energy $\gamma$ and also called surface tension. For a solid surface, the surface area $A$ can be increased either by creating a new surface (increasing the number of surface atoms, e.g., cleavage or plastic deformation with atoms moving to the surface) or by elastically straining the solid as a whole (keeping the number of surface atoms constant). The first possibility is the same as in a liquid and reflected by the term $\gamma$, whereas both right-hand terms must be considered in the second case.

To make this case of a strained surface a bit more clear, let us assume that the surface energy per atom remains unaffected by strain. Since we assume in this case that the number of atoms at the surface remains constant, this also means that the energy of the whole surface $A \gamma$ does not change with strain and the surface stress $g$ vanishes. In this case, the surface energy per area decreases proportionally to $A^{-1}$ and the two right-hand terms in Eq. (3.1) cancel.

There is also a more straightforward way to calculate surface stress based on the Lagrangian area $A_{L}$, i.e., the area of a surface in the unstrained state. We also define a Lagrangian surface energy $\gamma_{L}$, i.e., the energy per surface area in the unstrained state.
Using summation over repeated indices and assuming infinitesimally small strain, we may write

$$
\begin{aligned}
& A=A_{L}\left(1+\varepsilon_{\alpha \alpha}\right), \\
& \gamma_{L}=\gamma\left(1+\varepsilon_{\alpha \alpha}\right),
\end{aligned}
$$

and hence

$$
g_{\alpha \beta}=\frac{1}{A_{L}} \frac{\partial\left(A_{L} \gamma_{L}\right)}{\partial \varepsilon_{\alpha \beta}}=\frac{\partial \gamma_{L}}{\partial \varepsilon_{\alpha \beta}} .
$$

Since the Lagrangian surface energy $\gamma_{L}$ does not refer to a fixed area in space but rather to a fixed amount of surface atoms (unit area in the unstrained state), Eq. (3.4) immediately shows us that the surface stress vanishes if the surface energy per atom does not change with strain. If $g_{\alpha \beta}$ is nonzero, the surface atoms will try to achieve a state of lower energy by exerting a force onto the bulk below.

Unfortunately, it is not always clearly stated which of the two definitions of surface energy $\gamma$ or $\gamma_{L}$ is used. Whereas the former may seem more natural from the thermodynamic point of view, $\gamma_{L}$ is more helpful in atomic-scale considerations and therefore used in Ref. 9.

Since we are dealing with free surfaces (in the sense of elasticity theory), where the components of stress perpendicular to the surface vanish, surface strain can be fully characterized by the in-plane components, which are denoted by greek suffixes. Therefore, the surface stress tensor $g_{\alpha \beta}$ has two normal components $g_{11}$ and $g_{22}$ (taking the $x_{3}$ axis perpendicular to the surface) and one face-shear component $g_{12}$. As the tensor must obey the symmetry of the surface, the face shear component vanishes for the three low-index fcc surfaces if one axis (here $x_{1}$ ) is chosen along a close-packed direction in the surface and the remaining two components are equal for the (111) as well as the (100) surface.

Table II gives numerical results for the surface stress

TABLE II. Surface stress $g_{\alpha \beta}$ (in $\mathrm{eV} /$ atom), cohesive and atomic-sphere energy contributions to $g_{\alpha \beta}$,

\begin{tabular}{|c|c|c|c|c|}
\hline $\begin{array}{l}\text { Surface } \\
\text { strain direction }\end{array}$ & (111) & $(100)$ & {$[\overline{1} 10]$} & {$[001]$} \\
\hline$g_{\alpha \beta}$ EMT, relaxed, total & +0.46 & +0.54 & +0.70 & +0.38 \\
\hline$g_{\alpha \beta}$ EMT, relaxed, $E_{c}$ & +0.78 & +0.60 & +0.93 & +0.33 \\
\hline$g_{\alpha \beta}$ EMT, relaxed, $E_{\mathrm{AS}}$ & -0.32 & -0.06 & -0.23 & +0.05 \\
\hline$\Delta d_{12} / d_{\text {bulk }}$, EMT $(\%)$ & -1.8 & -2.9 & \multicolumn{2}{|c|}{-7.3} \\
\hline$g_{\alpha \beta}$ EMT, unrelaxed, total & +0.71 & +1.05 & +1.55 & +0.56 \\
\hline$g_{\alpha \beta \text { relaxed }}-g_{\alpha \beta \text { unrelaxed }}$, EMT & -0.25 & -0.51 & -0.85 & -0.18 \\
\hline$g_{\alpha \beta \text { relaxed }}-g_{\alpha \beta \text { unrelaxed }}$, Eq. (3.7) & -0.24 & -0.47 & -0.84 & -1.18 \\
\hline$g_{\alpha \beta} a b$ initio, unrelaxed & & $\begin{array}{l}+1.05 \\
-0.8\end{array}$ & & \\
\hline$\Delta d_{12} / d_{\text {bulk }}$, ab initio $(\%)$ & & $\begin{array}{l}-0.8 \\
+2.5 \pm 2.5^{\mathrm{b}}\end{array}$ & \multirow{2}{*}{\multicolumn{2}{|c|}{$\begin{array}{c}-6 \pm 2^{\mathrm{c}} \\
-5.1 \pm 1.5^{\mathrm{f}}\end{array}$}} \\
\hline$\Delta d_{12} / d_{\text {bulk }}$, experimental (\%) & $\begin{array}{r}0.0 \pm 0.3^{\mathrm{a}} \\
+1.3 \pm 1.3^{\mathrm{d}}\end{array}$ & $\begin{array}{l}+2.5 \pm 2.5^{\circ} \\
+3.1 \pm 1.5^{\mathrm{e}}\end{array}$ & & \\
\hline
\end{tabular}
and surface relaxation $\Delta d_{12} / d_{\text {bulk }}$ (in percent) for the low-index surfaces of Pd. For the (110) surface, the components $g_{11}$ and $g_{22}$ (for strains $\varepsilon_{\alpha \beta}$ along [110] and [001], respectively) are different. Ab initio results from Ref. 9 and experimental values of surface relaxation are given for comparison.

${ }^{\text {a}}$ Reference 57.

${ }^{\mathrm{b}}$ Reference 58.

${ }^{\mathrm{C}}$ Reference 59.
${ }^{\mathrm{d}}$ Reference 60.

${ }^{\mathrm{e}}$ Reference 61 .

${ }^{\mathrm{f}}$ Reference 62. 
$g_{\alpha \beta}$ of Pd calculated with effective-medium potentials We have included data on both unrelaxed and relaxed surfaces to facilitate comparison with the $a b$ initio result calculated for an unrelaxed surface. ${ }^{9}$ All values of $g_{\alpha \beta}$ are positive, indicating tensile stress. It is obvious that surface stress increases with the openness of the surface, i.e., from (111) to (110). This is the same trend as for surface relaxation. In the effective-medium theory both $g_{\alpha \beta}$ and relaxation have the same driving force, i.e., the tendency of an atom to increase its surrounding electronic density towards the optimum (higher) value, which it reaches in the bulk. The atoms in the more open surfaces are further from the optimum density, where the $E_{c}(n)$ function has higher slope and therefore can gain more energy by inward relaxation or by lateral contraction. ${ }^{21}$ This effect is counteracted by the mutual repulsion of the atoms described by the atomic-sphere energy $E_{\mathrm{AS}}$.

Unfortunately, we cannot easily test the accuracy of our data since there are no experimental results on surface stress of well-defined surfaces and only very few $a b$ initio data to compare with. Furthermore, $a b$ initio calculations of surface stress are rather sensitive to computational details and therefore not always accurate. ${ }^{5,11}$ The exact agreement of the stress values for $\operatorname{Pd}(100)$ calculated by EMT and an $a b$ initio method (Table II) is certainly more a coincidence than typical for the accuracy of either EMT or $a b$ initio calculations. We have also examined surface stress for other metals and found that EMT generally tends to underestimate surface stress compared to $a b$ initio calculations. For most metals investigated (Pd, Ag, Pt, and Au; ab initio data from Refs. 6 and 9) the deviation lies between a few percent and $40 \%$, whereas a more severe underestimation of both surface stress and inward relaxation of the (110) surface is found for the $3 d$ metals $\mathrm{Cu}$ and $\mathrm{Ni}$ (the $a b$ initio surface stress data are from Ref. 37).

Although it is possible to calculate surface stress for unrelaxed surfaces using Eq. (3.1) or (3.4) (and we have done so for comparison with $a b$ initio data), this is a merely hypothetical number and it can be far from the respective value of a real (relaxed) surface. In the effective-medium picture this is easily understandable since the driving force for stress is partly accommodated by relaxation and therefore surface stress is reduced by relaxation. For a more formal view of this problem, let us denote relaxation as $\rho$, which may be just one number (first layer relaxation) or include multilayer relaxations (then think of $\rho$ as a vector of different relaxation values and replace the derivative with the gradient symbol in the following equations). The surface energy then becomes a function of relaxation and strain $\gamma\left(\rho, \varepsilon_{\alpha \beta}\right)$ and the relaxed state is given by

$$
\left[\frac{\partial \gamma\left(\rho, \varepsilon_{\alpha \beta}\right)}{\partial \rho}\right]_{\varepsilon_{\alpha \beta} \mathrm{fixed}}=0,
$$

defining the relaxation $\rho\left(\varepsilon_{\alpha \beta}\right)$ as a function of strain. Therefore, the deviation of unrelaxed surface energy from the true (relaxed) value is of second order in $\rho$, i.e., rather small.

For the calculation of surface stress we will use La- grangian coordinates for simplicity. The surface stress for the relaxed surface is a sum of two terms

$$
\begin{aligned}
{\left[\frac{\partial \gamma_{L}\left(\varepsilon_{\alpha \beta}\right)}{\partial \varepsilon_{\alpha \beta}}\right]_{\rho=\rho\left(\varepsilon_{\alpha \beta}\right)}=} & {\left[\frac{\partial \gamma_{L}\left(\rho, \varepsilon_{\alpha \beta}\right)}{\partial \varepsilon_{\alpha \beta}}\right]_{\rho \text { fixed }} } \\
& +\left(\frac{\partial \gamma_{L}\left(\rho, \varepsilon_{\alpha \beta}\right)}{\partial \rho}\right]_{\varepsilon_{\alpha \beta} \text { fixed }} \frac{d \rho\left(\varepsilon_{\alpha \beta}\right)}{d \varepsilon_{\alpha \beta}},
\end{aligned}
$$

where the first term is the surface stress with fixed relaxation coordinates $\rho$ and the second is first order in $\left(\rho-\rho_{\text {relaxed }}\right)$. Thus, if one starts a computation of surface stress with the relaxed configuration, it is not necessary to relax the surface after applying strains, as the second term vanishes. If also the starting configuration is unrelaxed, however, the second term introduces a sizeable difference between relaxed and unrelaxed data, which is evident in Table II.

As most $a b$ initio values of surface stress published in the literature have been calculated for unrelaxed surfaces, it may be interesting whether one can calculate the true, i.e., the relaxed value, from continuum elastic theory. Assuming that only the first layer is relaxed and behaves like a bulk layer otherwise, the result is

$$
\begin{aligned}
g_{\alpha \beta_{\text {relaxed }}}-g_{\alpha \beta_{\text {unrelaxed }}} & =\left[\frac{\partial \gamma_{L}}{\partial \varepsilon_{\alpha \beta}}\right]_{\text {relaxed }}-\left[\frac{\partial \gamma_{L}}{\partial \varepsilon_{\alpha \beta}}\right]_{\text {unrelaxed }} \\
& =c_{\alpha \beta 33}^{\prime} \Delta d_{12},
\end{aligned}
$$

where $c_{i j k l}^{\prime}$ are the bulk elastic constants in the surface coordinate system (denoted by the prime), $\Delta d_{12}$ denotes the change of the first interlayer distance due to relaxation, and the surface is assumed to be perpendicular to $x_{3}$. Table II shows that this is a good approximation for the close-packed (111) and (100) surfaces, but completely fails for the [001] stress component of the (110) surface. This is not astonishing since a single (110) monolayer has no nearest neighbors along [001]; it therefore cannot be considered a layer on its own with regard to elasticity. These conclusions regarding the validity of Eq. (3.7) are confirmed by comparison with $a b$ initio calculations of surface stress differences for unrelaxed and relaxed $\operatorname{Ag}(100)$ (Ref. 37) and $\mathrm{Pb}(110){ }^{7}$

\section{B. Surface elasticity}

Similar to the first derivative of surface energy with respect to strain, i.e., surface stress, we can define the second derivative as

$$
C_{\alpha \beta \alpha^{\prime} \beta^{\prime}}^{s}=\frac{\partial^{2} \gamma_{L}}{\partial \varepsilon_{\alpha \beta} \partial \varepsilon_{\alpha^{\prime} \beta^{\prime}}}
$$

and we note that this quantity describes the difference in elastic behavior between a truncated continuum (in the sense of continuum elasticity theory) and the solid containing a real surface. From the viewpoint of bulk elasticity, it must be required that the components $\sigma_{3}$ of bulk 
stress perpendicular to the surface vanish at the surface, which means that only three independent strain components $\varepsilon_{\alpha \beta}\left(\varepsilon_{11}, \varepsilon_{12}\right.$, and $\left.\varepsilon_{22}\right)$ can be specified. The other strain components, including the expansion perpendicular to the surface $\varepsilon_{33}$, are functions of these. This also means that the interlayer distances freely expand or contract according to the in-plane strains and therefore surface elasticity must be calculated for a surface that is allowed to relax.

Numerical values for surface elasticity of $P d$ can be found in Table III (we did not include data related to face shear $\varepsilon_{12}$ since those have little practical significance for our purpose). The easiest way to interpret these data is by comparing them to bulk elasticity, if both values are expressed as energy per atom, i.e., multiplied with the atomic surface or atomic volume, respectively. It should be noted, however, that the second derivatives of elastic energy in the bulk

$$
C_{\alpha \beta \alpha^{\prime} \beta^{\prime}}^{b}=\left[\frac{\partial^{2} E}{\partial \varepsilon_{\alpha \beta^{2}} \partial \varepsilon_{\alpha^{\prime} \beta^{\prime}}}\right]_{\sigma_{3}=0}
$$

are not the bulk elastic constants but calculated by solving for the three dependent strain components $\left(\varepsilon_{13}, \varepsilon_{23}\right.$, and $\varepsilon_{33}$ ) and inserting them into the stress/strain ratios given by the bulk elastic constants [note that we use capital $C$ in Eq. (3.9), whereas we denote the bulk elastic constants with lowercase $c$ ].

Table III clearly shows that the more close-packed surfaces have nearly zero surface elasticity compared to the bulk, which means that the stress/strain ratio of the surface is almost the same as for a truncated elastic continuum. The (110) surface is significantly weaker than a corresponding bulk layer, especially upon strain in $x_{2}$, i.e., the [001] direction. Obviously this is a consequence of the trough-and-ridge structure of this surface, which reduces its resistance to strains perpendicular to the close-packed rows.

In Table III we also give the biaxial modulus, which we define as

TABLE III. Surface elasticity $C_{\alpha \beta \alpha^{\prime} \beta^{\prime}}^{s}$ for the low-index surfaces of Pd [Eq. (3.8)] and the corresponding bulk values $C_{\alpha \beta \alpha^{\prime} \beta^{\prime}}^{b}$ for zero strain perpendicular to the surface direction [Eq. (3.9)]. Furthermore, the biaxial modulus defined in Eqs. (3.10) and (3.11) is given. All values are in $\mathrm{eV} /$ atom, i.e., referred to the atomic surfaces or volumes for surface and bulk elasticity, respectively. For symmetry reasons, $C_{1111}=C_{2222}$ for the (111) and (100) surfaces.

\begin{tabular}{llcccc}
\hline \hline & $C_{1111}$ & $C_{1122}$ & $C_{2222}$ & $Y$ \\
\hline $\operatorname{Pd}(111)$ & $C_{\alpha \beta \alpha^{\prime} \beta^{\prime}}^{s}$ & -0.3 & +1.2 & & +0.9 \\
$\operatorname{Pd}(111)$ & $C_{\alpha \beta \alpha^{\prime} \beta^{\prime}}^{b}$ & 21 & 10 & & 31 \\
& & & & \\
$\operatorname{Pd}(100)$ & $C_{\alpha \beta \alpha^{\prime} \beta^{\prime}}^{s}$ & -2.3 & +2.0 & & -0.3 \\
$\operatorname{Pd}(100)$ & $C_{\alpha \beta \alpha^{\prime} \beta^{\prime}}^{b}$ & 16 & 0 & & 16 \\
& & & & \\
$\operatorname{Pd}(110)$ & $C_{\alpha \beta \alpha^{\prime} \beta^{\prime}}^{s}$ & -5.7 & -3.6 & -5.9 & -9.4 \\
$\operatorname{Pd}(110)$ & $C_{\alpha \beta \alpha^{\prime} \beta^{\prime}}^{b}$ & 22 & 8 & 10 & 24 \\
\hline \hline
\end{tabular}

TABLE IV. Relative changes of surface and bulk interlayer distance with in-plane strain for the low-index surfaces of Pd. For the (110) surface, the values for strains $\varepsilon_{11}$ and $\varepsilon_{22}$ (along [110] and [001], respectively) are different. First- and secondlayer relaxations of the unstrained surfaces (in percent) are also given.

\begin{tabular}{lcccc}
\hline \hline \multirow{2}{*}{$\begin{array}{l}\text { Surface } \\
\text { strain direction }\end{array}$} & \multirow{2}{*}{$(11)$} & $(100)$ & \multicolumn{2}{c}{$(110)$} \\
\hline$\partial \ln d_{12} / \partial \varepsilon_{\alpha \beta}$ & -0.57 & -0.86 & -0.97 & -1.00 \\
$\partial \ln d_{23} / \partial \varepsilon_{\alpha \beta}$ & -0.46 & -0.74 & -0.12 & -0.23 \\
$\partial \varepsilon_{33} / \partial \varepsilon_{\alpha \beta}=\partial \ln d_{\text {bulk }} / \partial \varepsilon_{\alpha \beta}$ & -0.47 & -0.75 & -0.42 & -0.59 \\
& & & & \\
$\Delta d_{12} / d_{\text {bulk }}(\%)$ & -1.8 & -2.9 & -7.3 \\
$\Delta d_{23} / d_{\text {bulk }}(\%)$ & +0.15 & +0.21 & +1.10 \\
\hline \hline
\end{tabular}

$$
\begin{aligned}
Y^{b} & =\frac{1}{2}\left[\frac{\partial^{2} E}{\partial \varepsilon^{2}}\right]_{\varepsilon_{11}=\varepsilon_{22}=\varepsilon, \sigma_{3}=0} \\
& =C_{1111}^{b} / 2+C_{1122}^{b}+C_{2222}^{b} / 2,
\end{aligned}
$$

i.e., the second-order increase of energy upon simultaneous compression along $x_{1}$ and $x_{2}$, and the respective value for the surface

$$
\begin{aligned}
Y^{s} & =\frac{1}{2}\left(\frac{\partial^{2} \gamma_{L}}{\partial \varepsilon^{2}}\right)_{\varepsilon_{11}=\varepsilon_{22}=\varepsilon, \sigma_{3}=0} \\
& =C_{1111}^{s} / 2+C_{1122}^{s}+C_{2222}^{s} / 2 .
\end{aligned}
$$

Being a sum of surface elastic constants, $Y^{S}$ also indicates hardly any change of elastic constants at the more closepacked surfaces with respect to the bulk.

Another interesting question concerns the change of relaxation with strain. As mentioned previously, the condition of a free surface implies that $\varepsilon_{33}$ cannot be freely specified but depends on the three independent strain components. It is clear that, due to transverse contraction, the derivative of interlayer distance or of $\varepsilon_{33}$ with respect to in-plane strain $\varepsilon_{11}$ or $\varepsilon_{22}$ is negative. Table IV further shows that the interlayer spacing of the top two monolayers reacts much more sensitively to lateral strain than in the bulk, whereas the distance between second and third layer changes less than the bulk interlayer distance.

Unfortunately, there are no experimental or ab initio data on surface elasticity to compare with our results. Thus we cannot determine the accuracy of our calculations, but we note that good agreement of EMT results and both bulk and surface phonon data determined experimentally has been found. ${ }^{20,38,39}$ This fact and the good agreement of the anisotropy of bulk elastic constants and other data mentioned previously indicate that EMT potentials are a good choice for the calculation of surface elasticity.

\section{STRESS AND STRAIN FOR INDIVIDUAL LAYERS: THE METHOD OF ARTIFICIAL ATOMS}

Whereas the derivatives of surface energy described in the preceding section can be only defined for a surface as 
a whole, there are many applications were data on stress and elasticity are needed for individual layers. A straightforward approach to this problem would be defining stress and elasticity as first and second derivatives of the energies of individual layers. It is clear that this cannot be done by $a b$ initio total-energy methods, where the energy cannot be partitioned between different atoms. In the effective-medium approach, only the cohesive energy $E_{c}$ can be unambiguously attributed to individual atoms, whereas the atomic-sphere term $E_{\text {AS }}$ stems from an overlap of electron density tails between two or more atoms. A conceptually consistent way of partitioning $E_{\mathrm{AS}}$ is attributing the contribution due to density tails of the other atoms reaching into the neutral sphere of a given atom to this atom. We have tried this approach, but the results were very different from the results obtained with artificial atoms described below. As the method of artificial atoms yields more direct answers to our questions, we will use this method and not try to find some indirect solution based on partitioning total energy between different layers.

In contrast to $a b$ initio methods, where the properties of atoms are in principle completely determined by ordinal number, atomic mass, and a few elementary constants, EMT allows us to tailor atoms with specific properties. For our purposes, we create artificial atoms with different size but otherwise unchanged properties, i.e., we multiply all parameters with the dimension of a length $\left(s_{0}\right)$ with a size factor $S$, and inverse lengths $\left(\lambda, \kappa, \eta_{2}\right)$ with $S^{-1}$. The energies $E_{0}$ and $V_{0}$ remain unchanged. The surface energy, the surface stress, and the bulk and surface elastic constants per atom (not per volume or per area) remain unchanged if the lattice constant of the artificial crystal is changed according to $S$.

If there is more than one kind of atom in a system, we also have to specify the electron density $n_{0}$ for the atoms. In principle we are free to chose whether we want to scale $n_{0}$ with $S^{-3}$ or keep it constant; this does not change the properties of the pure artificial element discussed above and depends on the question of interest. As our aim is mainly in the study of reconstructions (where the surface atoms are of the same kind as the bulk) and in segregation involving elements in the same column of the periodic system, we will keep the number of electrons per atom fixed, i.e., scale $n_{0}$.

Having created artificial atoms of different size, we may now compose an entire layer of atoms with a different size and calculate the total energy of such a slab. In the bulk of an unstrained crystal, any change of atomic size will cause stress energy and the energy will be lowest if the relative atomic size $S$ of the layer equals 1 . If a layer is under tensile stress, as surface layers usually are, larger atoms will be favored energetically, leading to a negative slope of $E(S)$ at $S=1$ and a minimum of energy at $S>1$. Furthermore, we can judge elasticity of a layer from the curvature of the $E(S)$ curves.

The results of these calculations for Pd are shown in Fig. 1 and Table V. For the more close-packed (111) and (100) surfaces, only the first layer is significantly different from the bulk, with sizable tensile stress, and a slightly lower curvature (elasticity) than a bulk layer. The curves
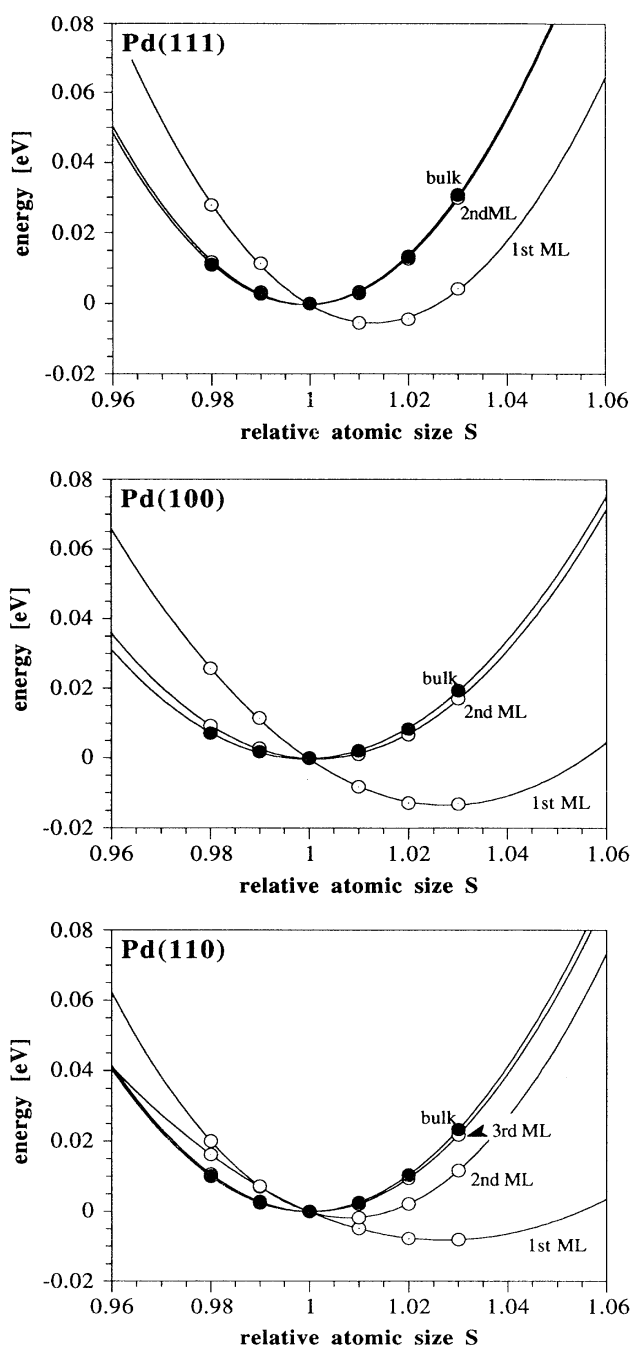

FIG. 1. Total energy as a function of atomic size for a crystal with a given layer made of artificial atoms with relative size $S$, but otherwise unchanged properties of $\mathbf{P d}$ (full line). All other layers are assumed to consist of normal Pd (relative size $S=1$ ). All lines are parabolic fits through the calculated data (open and filled circles are for surface and bulk layers, respectively).

TABLE V. Most favored atomic size the first three layers [minima of $E(S)$ in Fig. 1], compared to the respective value calculated from surface stress and surface elasticity assuming that only the first layer is different from the bulk. Furthermore, the second derivative of energy with respect to the size of artificial atoms in a single bulk layer is compared to the biaxial modulus (3.10). Energy and biaxial modulus are in $\mathrm{eV} /$ atom.

\begin{tabular}{lccc}
\hline \hline Surface & $(111)$ & $(100)$ & $(110)$ \\
\hline$S\left(E_{\min }\right)$, layer 1 & 1.014 & 1.029 & 1.029 \\
$S\left(E_{\min }\right)$, layer 2 & 1.001 & 1.002 & 1.008 \\
$S\left(E_{\min }\right)$, layer 3 & 1.000 & 1.000 & 1.001 \\
$S\left(E_{\min }\right)$, Eqs. (4.1) and $(4.2)$ & 1.015 & 1.037 & 1.037 \\
$\frac{1}{2} d^{2} E / d S^{2}$, bulk layer & 32 & 20 & 26 \\
$Y^{b}$ & 31 & 16 & 24 \\
\hline \hline
\end{tabular}


for the second layers are only slightly shifted to the right, indicating a small tensile stress and bulk elastic behavior. On the more open (110) surface, tensile stress is significant in the first two layers and the first layer is elastically much weaker (lower curvature) compared to the bulk.

With the artificial-atom approach, we have now seen how surface stress and elasticity is distributed between the different layers, but we have not determined welldefined elastic constants as we did for the surface as a whole. There is no possibility to discriminate between different strain directions - but there are a few quantities that we can compare between the macroscopic or thermodynamic quantities in Sec. III and the layerwise artificial-atom results.

One of the most interesting results to compare is the atomic size $S\left(E_{\min }\right)$ of the surface layer where the energy becomes a minimum and which we will call the most favored size for that layer. This quantity can be also calculated from surface stress and elasticity if we assume that only the first layer is different from the bulk and that its elastic energy $E$ is described by continuum mechanics with a strain offset $\varepsilon_{\alpha \beta}^{(0)}$ due to surface stress. Taking all elastic constants as quantities per atom (i.e., multiplied with the atomic volume or surface), we may write the stress/strain ratios of the first monolayer as $C^{b}-C^{s}$ and therefore the elastic energy of the first layer becomes

$$
\begin{aligned}
E_{L}= & \frac{1}{2}\left(C_{1111}^{b}-C_{1111}^{s}\right)\left(\varepsilon_{11}-\varepsilon_{11}^{(0)}\right)^{2} \\
& +\left(C_{1122}^{b}-C_{1122}^{s}\right)\left(\varepsilon_{11}-\varepsilon_{11}^{(0)}\right)\left(\varepsilon_{22}-\varepsilon_{22}^{(0)}\right) \\
& +\frac{1}{2}\left(C_{2222}^{b}-C_{2222}^{s}\right)\left(\varepsilon_{22}-\varepsilon_{22}^{(0)}\right)^{2} .
\end{aligned}
$$

Using this equation and the fact that $g_{\alpha \beta}=\partial E_{L} / \partial \varepsilon_{\alpha \beta}$ we may calculate the strain offsets $\varepsilon_{\alpha \beta}^{(0)}$ from surface stress $g_{\alpha \beta}$ and then get the isotropic strain $\varepsilon^{(0)}$ of minimum energy. We then assume that the most favored size for atoms in the surface layer exactly compensates for this isotropic strain, i.e.,

$$
S\left(E_{\min }\right)=\frac{1}{1+\varepsilon^{(0)}} .
$$

Table V shows that this approach, i.e., calculating the most favored atomic size for the topmost layer from surface stress and elasticity, overestimates the difference between first layer and the bulk. This is mainly due to the fact that surface stress located in deeper layers adds to total stress and hence to the difference of most favored size calculated therefrom. This argument alone cannot explain the discrepancy for the (100) surface, however, where deeper layers contribute only little to surface stress. For further insight to this problem, Table $\mathrm{V}$ also shows $Y^{b}$ (3.10) and $\frac{1}{2} d^{2} E / d S^{2}$ for a single layer of artificial atoms of size $S$ in the bulk. Whereas these two quantities should be identical if monoatomic bulk layers could be described by continuum mechanics, Table $\mathrm{V}$ tells us that the deviation is largest for the (100) orientation. We conclude that the deviation from continuum elasticity for a single atomic layer adds to the difference between most favored atomic size calculated from macro- scopic data and the layer-by-layer artificial atom approach.

\section{APPLICATION TO SURFACE SEGREGATION}

There are several quantities determining the difference between surface and bulk compositions in substitutional alloys, i.e., surface segregation. If we only want to determine which of the two constituents $A$ and $B$ of an alloy segregates or if we consider thermodynamic equilibrium at sufficiently low temperatures, where we need not consider entropy, we may restrict ourselves to the energetics of segregation. The main contributions to segregation energy in substitutional alloys are, in this case, (i) the difference of surface energies of the two elements, (ii) mixing energy (roughly speaking the difference in energy between two $A-B$ bonds and $A-A$ plus $B-B$ bonds), and (iii) the size effect, i.e., energetic contributions due to different size. Whereas the first two quantities are well understood, we will concentrate on the size effect here. To simplify our reasoning, in the following we will often mention the energy changes caused by size effect only, even though other effects must be taken into account.

In thermodynamic theories of surface segregation, surface stress is often neglected. For equal element concentrations in the bulk and at the surface, the only difference of elastic energy then comes from the difference in elasticity, i.e., the surface is assumed to be weaker than the bulk and its resistance to incorporating larger or smaller atoms hence lower. This means that substitutional impurity (minority) atoms, whether they are larger or smaller than the majority atoms, will always tend to segregate to the surface to reduce the strain energy caused by the impurity. This effect may be counteracted by surface energy differences, mixing energies, and entropy. Deviations from this symmetry with respect to atomic size difference come from nonlinear elasticity, but they do not change the situation qualitatively. ${ }^{18,19}$

Whereas this simple approach does not predict any size effect in a substitutional disordered alloy of two atomic species with different size and $\approx 50 \%$ concentration, the situation changes qualitatively if we include the tensile stress usually present at surfaces. It is clear that tensile surface stress will favor larger atoms at the surface, a fact directly reflected by the most favored atomic size $S\left(E_{\min }\right)$ described in Sec. IV.

For a more quantitative treatment, let us consider a kind of mean-field approach, i.e., assume that the atomic sizes of all atoms within a layer are equal, neglecting strain fields of individual atoms. This is a good approximation for a disordered system and concentrations around $50 \%$. We may then directly employ our study of individual layers composed of artificial atoms and take the results in Fig. 1 as the size effect of segregation energy.

As Fig. 1 shows, the total energy of a system can be reduced by moving larger atoms to the surface, assuming that the elastic energy in the bulk does not change. This assumption is valid around $50 \%$ bulk concentration; for other bulk concentrations bulk strain will be caused by moving missized atoms into the bulk.

Considering that we are dealing with elastic energies, 
the energies associated with the surface tension are 'quite substantial (Table VI): If we assume Pd atoms with a size difference of $11 \%( \pm 5.5 \%$ with respect to the bulk) we get for the (111) surface a size effect energy of $0.09 \mathrm{eV}$ per atom. For the (100) surface the value is even larger due to the higher surface stress, but it is lower for the (110) surface because the surface layer is elastically much weaker and because the surface stress is distributed between two layers.

As $\mathrm{Pd}$ is situated between $\mathrm{Pt}$ and $\mathrm{Ni}$ in the Periodic Table and as the size mismatch between $\mathrm{Pt}$ and $\mathrm{Ni}$ is $11 \%$, we can directly employ these data on the study of the size effect in segregation in the Pt-Ni system. The normal $\mathrm{Pd}$ atoms stand for the average bulk concentration of $50 \% \mathrm{Pt}$ and $\mathrm{Ni}$ and the size difference of $\pm 5.5 \%$ is the difference between these and pure $\mathrm{Pt}$ or $\mathrm{Ni}$, respectively.

With the assumptions described above, we may write the segregation energy for the substitution of a $\mathrm{Ni}$ atom by a $\mathrm{Pt}$ atom as a sum of surface energy and size effect contributions

$$
E_{\text {segr }}=\gamma_{\mathrm{Ni}}-\gamma_{\mathrm{Pt}}+E_{\text {size eff }},
$$

where the surface energies $\gamma_{\mathrm{Ni}}$ and $\gamma_{\mathrm{Pt}}$ have to be taken as quantities per atom. We define $E_{\text {size eff }}=E(0.945)$ $-E(1.055)$ for a given layer as the difference between the energy $E(S)$ of the layer made of smaller atoms $(-5.5 \%)$ and larger $(+5.5 \%)$ atoms.

For the (110) surface, where both the first and the second monolayer contribute substantially to the size effect, we must know the actual concentration profile to determine the size effect in both monolayers. This can be done by introducing the effect of chemical order to the energetics, but for simplicity we take the result of such calculations here, which yield a strongly oscillating segregation profile, i.e., alternating layers of $\mathrm{Pt}$ and $\mathrm{Ni}$ enrichment. ${ }^{40,41}$ We therefore assume that any Ni enrichment in the first monolayer is accompanied by $\mathrm{Pt}$ enrichment of the same amount in the second layer and therefore the total size effect contribution can be calculated as

$$
E_{\text {size eff }}=E_{\text {size eff 1 }}-E_{\text {size eff } 2} \text {. }
$$

Results of these calculations can be found in Table VI. Since the difference of surface energies between $\mathrm{Pt}$ and $\mathrm{Ni}$ is small, we see that the size effect plays an important role in this system. For the (111) surface, one would predict Ni segregation from the difference of surface energies, but the size effect favors the larger Pt atoms due to tensile surface stress. This leads to weak Pt segregation in our calculation, but we should note that any increase of the surface Pt concentration will also increase the tensile surface stress due to the higher surface stress of Pt compared to $\mathrm{Ni}$ or Pd (Ref. 9) and thereby enhance the effect. Pt segregation on $\mathrm{Pt}_{x} \mathrm{Ni}_{1-x}(111)$ surfaces is confirmed experimentally. $42-45$

For the (100) surface we find $\mathrm{Ni}$ segregation since the size effect is not large enough to reverse the surface energy difference. This value cannot be directly compared to experiment, however, since $\mathrm{Pt}_{x} \mathrm{Ni}_{1-x}(100)$ surfaces are known to reconstruct at $\mathrm{Pt}$ concentrations above a few percent. ${ }^{43,46,47}$ Since these experiments show that the tendency to reconstruct increases with the amount of surface $\mathrm{Pt}$ and the reconstructions lower the surface energy, $\mathrm{Pt}$ segregation will be favored by the reconstructions. Unfortunately, reconstruction energetics are not well known, but on the order of magnitude of $0.05 \mathrm{eV}$ per $\mathrm{Pt}$ atom. ${ }^{9}$ We therefore cannot decide about the resulting energetics and predict whether $\mathrm{Pt}$ or $\mathrm{Ni}$ segregates. Experimentally, $\mathrm{Pt}$ segregation is found on all $\mathrm{Pt}_{x} \mathrm{Ni}_{1-x}(100)$ surfaces studied so far. ${ }^{43,48,49}$

On the (110) surface the resulting size effect is smallest due to the elastic weakness of the first monolayer and the counteracting effect of the second; therefore strong $\mathrm{Ni}$ segregation should be expected. This result as well as the $\mathrm{Pt}$ enrichment in the second monolayer mentioned earlier have been confirmed experimentally. ${ }^{43,49-51}$

TABLE VI. Surface energies and their differences for Pt and Ni low-index faces from self-consistent calculations, and size effect energy in the first two monolayers $E(0.945)-E(1.055)$ for Pd atoms with $\pm 5.5 \%$ size differences, according to the size mismatch of $\mathrm{Pt}$ and $\mathrm{Ni}$ on $(\approx 50 \%) \mathrm{PtNi}$ alloy. Size effect energies calculated from interpolation between $\mathrm{Pt}$ and $\mathrm{Ni}$ (Ref. 52) are also included. The segregation energy $\Delta E_{\text {segr }}$ favors $\mathrm{Pt}$ segregation if positive, otherwise $\mathrm{Ni}$ segregation. All values are in $\mathrm{eV} /$ atom and all surfaces are assumed to be unreconstructed for the calculations.

\begin{tabular}{lccccccc}
\hline \hline & $\begin{array}{c}\text { Theory } \\
\gamma_{\mathrm{Ni}}\end{array}$ & $\gamma_{\mathrm{Pt}}$ & $\gamma_{\mathrm{Ni}-} \gamma_{\mathrm{Pt}}$ & $\Delta E_{\text {size eff 1 }}$ & $\Delta E_{\text {size eff 2 }}$ & $\Delta E_{\text {segr }}$ & $\begin{array}{r}\text { Experiment } \\
\text { segregating } \\
\text { element }\end{array}$ \\
\hline$(111)$ & $0.86^{\mathrm{a}}$ & $0.95^{\mathrm{a}}$ & -0.07 & 0.09 & & +0.02 & $\mathrm{Pt}$ \\
& $0.88^{\mathrm{b}, \mathrm{c}}$ & $0.98^{\mathrm{b}}$ & (average) & & & & \\
& $0.90^{\mathrm{b}}$ & $0.91^{\mathrm{e}}$ & & $0.15^{\mathrm{g}}$ & & & \\
$(100)$ & $0.99^{\mathrm{a}}$ & $1.18^{\mathrm{a}}$ & -0.17 & 0.11 & & -0.06 & $\mathrm{Pt}^{\mathrm{h}}$ \\
& $1.06^{\mathrm{d}}$ & $1.21^{\mathrm{f}}$ & $($ average) & $0.13^{\mathrm{g}}$ & & & \\
$(110)$ & $1.47^{\mathrm{a}}$ & $1.65^{\mathrm{a}}$ & -0.18 & 0.07 & 0.05 & -0.16 & $\mathrm{Ni}$ \\
& & & & $0.09^{\mathrm{g}}$ & $0.06^{\mathrm{g}}$ & & \\
\hline \hline
\end{tabular}

${ }^{\text {a}}$ Reference 41.

${ }^{\mathrm{b}}$ Reference 63.

${ }^{c}$ Ferromagnetic.

${ }^{\mathrm{d}}$ Reference 37.
'Reference 6.

${ }^{f}$ Reference 9.

${ }^{8}$ Reference 52.

${ }^{\mathrm{h}}$ Reconstructs. 
We do not claim quantitative exactness of the segregation energies calculated here; both surface energies and the approximation of the size effect by artificial big and small Pd atoms are not sufficiently accurate. Nevertheless, the trend of different segregating components- $\mathbf{P t}$ on the more close-packed and $\mathrm{Ni}$ on the more open surface - has been well described by the balance between surface energy differences and size effect. This confirms the influence of the size effect caused by surface stress for the face-dependent segregation of Pt-Ni alloys, which has been proposed earlier. ${ }^{52,53}$ We also note that the size effect energies from Ref. 52, which have been obtained by interpolation between tight-binding results for $\mathrm{Pt}$ and $\mathrm{Ni}$, are in reasonable agreement with our calculations presented here, in spite of the totally different approach (Table VI).

\section{CONCLUSIONS}

We have used effective-medium potentials to study surface stress, surface elasticity, and related phenomena for low-index surfaces of fcc metals and given data for Pd. In agreement with other studies, we have found tensile surface stress increasing with the openness of the surface. In the effective-medium theory, surface stress can be explained by the tendency of atoms to move towards their optimum electronic density.

The second derivatives of surface energy with respect to strain, which describe surface elasticity, were also studied. It was found that the more closely packed surfaces have essentially the same elasticity as a truncated continuum, whereas the (110) surface is significantly weaker, especially for strain along [001].

For attributing surface stress and elasticity to individual atomic layers, we have introduced the method of artificial atoms. Using artificial atoms with different size, but otherwise the same properties as the normal material, we can probe stress and elasticity of individual layers. For the (111) and (100) surfaces, the second monolayer can be regarded as bulklike except for its slight tensile stress, whereas significant stress was found in the second monolayer of a (110) surface. On all surfaces, significant differences between surface and bulk elasticity (with regard to compression or expansion) are found in the first monolayer only.

We have also compared several data calculated by effective-medium theory with continuum elasticity theory applied to individual atomic layers. It was generally found that continuum elasticity is a good approximation for the close-packed (111) oriented layers, whereas it is less accurate for (100) and completely fails for (110) layers strained along [001]. One remarkable exception is the dependence of interlayer distance between the first and second surface layers on lateral compressive or tensile strain. This quantity, $\partial \ln d_{12} / \partial \varepsilon_{\alpha \beta}$, is significantly larger than the respective bulk given by elasticity theory even for close-packed surfaces.

The results of the method of artificial atoms can be directly used to study the size effect in surface segregation. We have shown that the face-dependent segregation of Pt-Ni alloys is due to the size effect energy, which is caused by surface stress and the surface elasticity. For the more closely-packed surfaces (111) and (100), tensile surface stress significantly favors the larger $\mathrm{Pt}$ atoms in the first monolayer, whereas the effect of surface stress is weak on the (110) surface, where stress is distributed between the first and the second monolayer and the size effect in the first monolayer is reduced by surface elasticity.

A problem similar to surface segregation is the formation of surface alloys, which has become a wellestablished phenomenon in recent years. Again we might expect that surface stress and surface elasticity play an important role since they will usually favor atoms larger than the substrate atoms to be incorporated. Indeed, recent studies have shown the formation of surface alloys in such cases with even very large size difference, such as $\mathrm{Pb} / \mathrm{Cu}$ (Refs. 54 and 55 ) and $\mathrm{Na} / \mathrm{Al},{ }^{56}$ whereas surface alloys with atoms smaller than the bulk material are rare. We therefore conclude that the stress and size effects should be always kept in mind when dealing with chemically heterogeneous surfaces.

\section{ACKNOWLEDGMENTS}

We would like to thank C. Engdahl, P. Kratzer, and S. Takizawa for many interesting discussions. This work was supported by the Fonds zur Förderung der Wissenschaftlichen Forschung (Austrian Science Foundation) under Projects Nos. S6204 and S6201 and by the Hochschuljubiläumsstiftung der Stadt Wien. The Center of Atomic-scale Materials Physics is sponsored by the Danish National Research Foundation.
"FAX: + 431 5864203; electronic address:

schmid@ eapv38.tuwien.ac.at

${ }^{1}$ Ch. Wöll, S. Chiang, R. J. Wilson, and P. H. Lippel, Phys. Rev. B 39, 7988 (1989).

2J. V. Barth, H. Brune, G. Ertl, and R. J. Behm, Phys. Rev. B 42, 9307 (1990).

${ }^{3}$ K. G. Huang, Doon Gibbs, D. M. Zehner, A. R. Sandy, and S. G. J. Mochrie, Phys. Rev. Lett. 65, 3313 (1990); A. R. Sandy, S. G. J. Mochrie, D. M. Zehner, K. G. Huang, and Doon Gibbs, Phys. Rev. B 43, 4667 (1991).

${ }^{4}$ R. J. Needs, Phys. Rev. Lett. 58, 53 (1987); R. J. Needs and M.
J. Godfrey, Phys. Scr. T19, 391 (1987).

${ }^{5}$ R. J. Needs and M. J. Godfrey, Phys. Rev. B 42, 10933 (1990).

${ }^{6}$ R. J. Needs and M. Mansfield, J. Phys. Condens. Matter 1, 7555 (1989).

${ }^{7}$ M. Mansfield and R. J. Needs, Phys. Rev. B 43, 8829 (1991).

${ }^{8}$ D. Vanderbilt, Phys. Rev. Lett. 59, 1456 (1987).

${ }^{9}$ V. Fiorentini, M. Methfessel, and M. Scheffler, Phys. Rev. Lett. 71, 1051 (1993).

10J. F. Annett and J. E. Inglesfield, J. Phys. Condens. Matter 1, 3645 (1989).

${ }^{11}$ P. J. Feibelman, Phys. Rev. B 50, 1908 (1994). 
${ }^{12}$ G. J. Ackland and M. W. Finnis, Philos. Mag. A 54, 301 (1986).

${ }^{13}$ B. W. Dodson, Phys. Rev. Lett. 60, 2288 (1988).

${ }^{14}$ D. Wolf, Surf. Sci. 226, 389 (1990).

${ }^{15}$ Surface Phonons, edited by W. Kress and F. W. de Wette (Springer, Berlin, 1991).

${ }^{16} \mathrm{G}$. Benedek and J. P. Toennies, Surf. Sci. 299/300, 587 (1994).

${ }^{17}$ R. F. Wallis, Surf. Sci. 299/300, 612 (1994).

${ }^{18}$ F. F. Abraham, N.-H. Tsai, and G. M. Pound, Surf. Sci. 83, 406 (1979).

${ }^{19}$ F. F. Abraham and C. R. Brundle, J. Vac. Sci. Technol. 18, 506 (1981).

${ }^{20}$ K. W. Jacobsen, J. K. Nørskov, and M. J. Puska, Phys. Rev. B 35, 7423 (1987).

${ }^{21}$ K. W. Jacobsen, Comments Condens. Matter Phys. 14, 129 (1988).

22J. K. Nørskov, K. W. Jacobsen, P. Stoltze, and L. B. Hansen, Surf. Sci. 283, 277 (1993).

${ }^{23}$ P. Stoltze, J. Phys. Condens. Matter 6, 9495 (1994).

${ }^{24}$ K. W. Jacobsen, P. Stoltze, and J. K. Nørskov (unpublished).

${ }^{25}$ O. B. Christensen, K. W. Jacobsen, J. K. Nørskov, and M. Manninen, Phys. Rev. Lett. 66, 2219 (1991).

${ }^{26}$ L. Hansen, P. Stoltze, K. W. Jacobsen, and J. F. Nørskov, Phys. Rev. B 44, 6523 (1991).

${ }^{27}$ J. H. Rose, J. Ferrante, and R. J. Smith, Phys. Rev. Lett. 47, 675 (1981).

${ }^{28}$ J. Ferrante, R. J. Smith, and J. H. Rose, Phys. Rev. Lett. 50, 1385 (1983).

${ }^{29}$ J. H. Rose, R. J. Smith, F. Guinea, and J. Ferrante, Phys. Rev. B 29, 2963 (1984).

${ }^{30}$ F. Ercolessi, E. Tosatti, and M. Parinello, Phys. Rev. Lett. 57, 719 (1986).

${ }^{31}$ M. W. Finnis and J. S. Sinclair, Philos. Mag. A 50, 45 (1984); 53, 161 (1986).

${ }^{32}$ M. S. Daw and M. I. Baskes, Phys. Rev. Lett. 50, 1285 (1983); Phys. Rev. B 29, 6443 (1984).

${ }^{33}$ R. A. Johnson, Phys. Rev. B 37, 3924 (1988).

${ }^{34}$ P. Stoltze, J. Chem. Phys. 92, 6306 (1990).

${ }^{35}$ R. Shuttleworth, Proc. Phys. Soc. London Sect. A 63, 444 (1950).

${ }^{36}$ R. C. Cammarata, Prog. Surf. Sci. 46, 1 (1994).

${ }^{37} \mathrm{~V}$. Fiorentini (private communication).

${ }^{38}$ P. D. Ditlevsen and J. K. Nørskov, Surf. Sci. 254, 261 (1991).

${ }^{39}$ P. D. Ditlevsen and J. K. Nørskov, J. Electron Spectrosc. Relat. Phenom. 54/55, 237 (1990).

${ }^{40}$ B. Legrand, G. Tréglia, and F. Ducastelle, Phys. Rev. B 41, 4422 (1990).
${ }^{41}$ I. A. Abrikosov, A. V. Ruban, H. L. Skriver, and B. Johansson, Phys. Rev. B 50, 2039 (1994).

${ }^{42}$ Y. Gauthier, Y. Joly, R. Baudoing, and J. Rundgren, Phys. Rev. B 31, 6216 (1985).

${ }^{43}$ Y. Gauthier and R. Baudoing, in Surface Segregation Phenomena, edited by P. A. Dowben and A. Miller (Chemical Rubber, Boca Raton, FL, 1990), p. 169.

${ }^{44}$ S. Deckers, F. H. P. M. Habraken, W. F. van der Weg, A. W. Denier van der Gon, B. Pluis, J. F. van der Veen, and R. Baudoing, Phys. Rev. B 42, 3253 (1990).

${ }^{45}$ P. Weigand, W. Hofer, and P. Varga, Surf. Sci. 287/288, 350 (1993).

${ }^{46}$ M. Schmid, A. Biedermann, S. D. Böhmig, P. Weigand, and P. Varga, Surf. Sci. 318, 289 (1994).

${ }^{47}$ Y. Gauthier, R. Baudoing-Savois, J. Rundgren, M. Hammar, and M. Gothelid, Surf. Sci. 327, 100 (1995).

${ }^{48}$ Y. Gauthier, W. Hoffmann, and M. Wuttig, Surf. Sci. 233, 239 (1990).

${ }^{49}$ P. Weigand, B. Jelinek, W. Hofer, and P. Varga, Surf. Sci. 301, 306 (1994).

${ }^{50}$ Y. Gauthier, R. Baudoing, M. Lundberg, and J. Rundgren, Phys. Rev. B 35, 7867 (1987).

${ }^{51}$ P. Weigand, B. Jelinek, W. Hofer, and P. Varga, Surf. Sci. 295, 57 (1993).

${ }^{52}$ G. Tréglia and B. Legrand, Phys. Rev. B 35, 4338 (1987).

${ }^{53}$ H. Stadler, W. Hofer, M. Schmid, and P. Varga, Surf. Sci. 287/288, 366 (1993).

${ }^{54}$ C. Nagl, O. Haller, E. Platzgummer, M. Schmid, and P. Varga, Surf. Sci. 321, 237 (1994).

${ }^{55}$ C. Nagl, E. Platzgummer, O. Haller, M. Schmid, and P. Varga, Surf. Sci. (to be published).

${ }^{56} \mathrm{~J}$. Neugebauer and M. Scheffler, Phys. Rev. B 48, 16067 (9192).

${ }^{57}$ Y. Kuk, L.C. Feldman, and P. J. Silverman, Phys. Rev. Lett. 50, 511 (1983).

${ }^{58}$ R. J. Behm, K. Christmann, G. Ertl, and M. A. Van Hove, J. Chem. Phys. 73, 2984 (1980).

${ }^{59}$ C. J. Barnes, M. Q. Ding, M. Lindroos, R. D. Diehl, and D. A. King, Surf. Sci. 162, 59 (1985).

${ }^{60}$ H. Ohtani, M. A. Van Hove, and G. A. Somorjai, Surf. Sci. 187, 372 (1987).

${ }^{61}$ J. Quinn, Y. S. Li, D. Tian, H. Li, F. Jona, and P. M. Marcus, Phys. Rev. B 42, 11348 (1990).

${ }^{62}$ M. Skottke, R. J. Behm, G. Ertl, V. Penka, and W. Moritz, J. Chem. Phys. 87, 6191 (1987).

${ }^{63}$ H. L. Skriver and N. M. Rosengaard, Phys. Rev. B 46, 7157 (1992). 